Hou Yu Zhao*

\title{
Pseudo almost periodic solutions for a class of differential equation with delays depending on state
}

https://doi.org/10.1515/anona-2020-0049

Received February 18, 2019; accepted October 4, 2019.

Abstract: In this paper, the exponential dichotomy, and Tikhonov and Banach fixed point theorems are used to study the existence and uniqueness of pseudo almost periodic solutions of a class of iterative functional differential equations of the form

$$
x^{\prime}(t)=\sum_{n=1}^{k} \sum_{l=1}^{\infty} C_{l, n}(t)\left(x^{[n]}(t)\right)^{l}+G(t),
$$

where $x^{[n]}(t)$ denotes $n$th iterate of $x(t)$.

Keywords: Iterative differential equation, pseudo almost periodic solutions, fixed point theorem

MSC: 39B12, 39B82

\section{Introduction}

Delay differential equation of the form

$$
x^{\prime}(t)=f\left(t, x(t), x\left(t-\tau_{1}(t)\right), \ldots, x\left(t-\tau_{k}(t)\right)\right)
$$

has been discussed in [1, 2]. In particularly, the delay functions $\tau_{j}(t), j=0,1, \ldots, k$ depend not only on unknown function, but also state, the delay functions $\tau_{j}(t, x(t)), j=0,1, \ldots, k$ have been studied in many literatures. In [3], Cooke pointed out that it is highly desirable to establish the existence and stability properties of periodic solutions for equations of the form

$$
x^{\prime}(t)+a x(t-h(t, x(t)))=F(t)
$$

in which the lag $h(t, x(t))$ implicitly involves $x(t)$. Si and Wang [4] discussed the smooth solutions of equation of

$$
x^{\prime}(t)=\lambda_{1} x(t)+\lambda_{2} x^{[2]}(t)+\ldots+\lambda_{n} x^{[n]}(t)+f(t),
$$

where $x^{[1]}(t)=x(t), x^{[2]}(t)=x(x(t)), x^{[3]}(t)=x(x(x(t))), \ldots$, i.e., $x^{[i]}(t)$ denotes $i$ th iterate of $x(t), i=$ $1,2, \ldots, n$. Later, by Schröder transformation, Liu and Si [5] considered the analytic solutions of the form

$$
x^{\prime}(t)=\sum_{n=0}^{k} \sum_{l=1}^{\infty} C_{l, n}(t)\left(x^{[n]}(t)\right)^{l}+G(t),
$$

*Corresponding Author: Hou Yu Zhao, School of Mathematics, Chongqing Normal University, Chongqing 401331, P.R. China, E-mail: houyu19@gmail.com 
where $x^{[n]}(t)$ denotes $n$th iterate of $x(t), n=0,1,2, \ldots, k$. Recently, In [6], Zhao and Fečkan studied the periodic solution of

$$
\chi^{\prime}(t)=\sum_{n=1}^{k} \sum_{l=1}^{\infty} C_{l, n}(t)\left(x^{[n]}(t)\right)^{l}+G(t)
$$

where $x^{[n]}(t)$ denotes $n$th iterate of $x(t), n=1,2, \ldots, k$. For some various properties of solutions for several iterative functional differential equations, we refer the interested reader to [7]-[11].

On the other hand, the existence of pseudo almost periodic solutions is among the most attractive topics in qualitative theory of differential equations due to their applications, especially in biology, economics and physics [12]-[16]. As pointed out by Ait Das and Ezzinbi [13], it is an interesting thing to study the pseudo almost periodic systems with delays. It is obviously that iterative functional differential equations are special type state-dependent delay differential equations. In [17], Liu pointed that the properties of the almost periodic functions do not always hold in the set of pseudo almost periodic functions and given an example: when $x(t)$ is a pseudo almost periodic function, $x(x(t))$ may not be a pseudo almost periodic function. To the best of our knowledge, there are few results about pseudo almost periodic solutions for iterative functional differential equations except [17], [18] and [19].

In the present work, we propose an existence result for pseudo almost periodic solutions of Eq (1.1) by using Tikhonov fixed theorem. Uniqueness of the solution is achieved by Banach fixed point theorem.

This paper is organized as follows. In Section 2 we give some notes and establish the main existence result. In Section 3, we show that (1.1) has a unique pseudo almost periodic solution under some suitable conditions. Furthermore, we prove the stability depend on $C_{l, n}$ and $G$. In Section 4, we present an example to illustrate the theory. Related problems are also studied in [20].

\section{Existence result}

In this section, the existence of pseudo almost periodic solutions of equation (1.1) will be studied. Throughout this paper, it will be assumed that

(H) $C_{1,1}(t): \mathbb{R} \rightarrow(-\infty, 0)$ is an almost periodic function, $C_{l, n}(t): \mathbb{R} \rightarrow \mathbb{R}$ are pseudo almost periodic functions, and

$$
C_{1,1}^{+}=\sup _{t \in \mathbb{R}} C_{1,1}(t)<0, C_{l, n}^{+}=\sup _{t \in \mathbb{R}}\left|C_{l, n}(t)\right|, G^{+}=\sup _{t \in \mathbb{R}}|G(t)|,
$$

where $l=1,2, \ldots ; n=2, \ldots, k$.

For convenience, we will use $C(\mathbb{R}, \mathbb{R})$ to denote the set of all continuous functions from $\mathbb{R}$ to $\mathbb{R}$ endowed with the usual metric $d(f, g)=\sum_{m=1}^{\infty} 2^{-m} \frac{\|f-g\|_{m}}{1+\|f-g\|_{m}}$ for $\|f-g\|_{m}=\max _{t \in[-m, m]}|f(t)-g(t)|$, so the topology on $C(\mathbb{R}, \mathbb{R})$ is the uniform convergence on each compact intervals of $\mathbb{R}$. We also consider the set $B C(\mathbb{R}, \mathbb{R})$ of all bounded and continuous functions from $\mathbb{R}$ to $\mathbb{R}$ with the norm $\|f\|=\sup _{t \in \mathbb{R}}|f(t)|$, so the topology on $B C(\mathbb{R}, \mathbb{R})$ is the uniform convergence on $\mathbb{R}$. Denote by $A P(\mathbb{R}, \mathbb{R})$ the set of all almost periodic functions from $\mathbb{R}$ to $\mathbb{R}$. Define the set

$$
P A P_{0}(\mathbb{R}, \mathbb{R})=\left\{g \in B C(\mathbb{R}, \mathbb{R})\left|\lim _{T \rightarrow+\infty} \frac{1}{2 T} \int_{-T}^{T}\right| g(t) \mid d t=0\right\} .
$$

A function $\varphi \in B C(\mathbb{R}, \mathbb{R})$ is called pseudo almost periodic if it can be expressed as $\varphi=h+g$, where $h \in$ $A P(\mathbb{R}, \mathbb{R})$ and $g \in P A P_{0}(\mathbb{R}, \mathbb{R})$. The collection of such functions will be denoted by $P A P(\mathbb{R}, \mathbb{R})$. In particular, $(P A P(\mathbb{R}, \mathbb{R}),\|\cdot\|)$ is a Banach space [21]. For $M, L>0$, define

$$
\begin{gathered}
B_{C}(M, L)=\left\{\varphi \in C(\mathbb{R}, \mathbb{R})|| \varphi(t)|\leq M,| \varphi\left(t_{2}\right)-\varphi\left(t_{1}\right)|\leq L| t_{2}-t_{1} \mid,\right. \\
\left.\quad \text { for all } t, t_{1}, t_{2} \in \mathbb{R}\right\},
\end{gathered}
$$




$$
B_{S}(M, L)=S(\mathbb{R}, \mathbb{R}) \cap B_{C}(M, L), \quad S \in\left\{P A P, A P, P A P_{0}\right\} .
$$

From [17], it is easy to see that $B_{S}(M, L), S \in\left\{P A P, A P, P A P_{0}, C\right\}$ are closed convex and bounded subsets of $B C(\mathbb{R}, \mathbb{R})$ and $C(\mathbb{R}, \mathbb{R})$, respectively. Furthermore, by the Arzelá-Ascoli theorem, the subset $B_{C}(M, L)$ is compact in $C(\mathbb{R}, \mathbb{R})$.

On the other hand, the subset $B_{C}(M, L)$ is not precompact in $B C(\mathbb{R}, \mathbb{R})$, since a sequence $\left\{M \operatorname{sech} \frac{L(t+n)}{M}\right\}_{n=1}^{\infty} \in B_{C}(M, L)$ has no a convergent subsequence in $B C(\mathbb{R}, \mathbb{R})$. As in [19], we have following Theorem.

Theorem 2.1. The subsets $B_{S}(M, L), S \in\left\{P A P, A P, P A P_{0}, C\right\}$ are not precompact in $B C(\mathbb{R}, \mathbb{R})$. They are precompact in $C(\mathbb{R}, \mathbb{R})$. Furthermore, $B_{A P}(M, L)$ and $B_{P A P_{0}}(M, L)$ are just precompact in $C(\mathbb{R}, \mathbb{R})$, while $B_{C}(M, L)$ is compact in $C(\mathbb{R}, \mathbb{R})$.

So (non-)compactness of $B_{P A P}(M, L)$ in $C(R, R)$ is still open.

Finally, we recall Tikhonov fixed point theorem.

Theorem 2.2. (Tikhonov) Let $\Omega$ be a non-empty compact convex subset of a locally convex topological vector space $X$. Then any continuous function $A: \Omega \rightarrow \Omega$ has a fixed point.

Now we apply Theorem 2.2. Let $X$ be either $P A P(\mathbb{R}, \mathbb{R})$ or $B C(\mathbb{R}, \mathbb{R})$ but fixed. If $\varphi \in X$, from Corollary 5.4 in [21], it follows $\varphi^{[2]} \in X$. Consider an auxiliary equation

$$
x^{\prime}(t)=C_{1,1}(t) x(t)+\sum_{n=2}^{k} C_{1, n}(t) \varphi^{[n]}(t)+\sum_{n=1}^{k} \sum_{l=2}^{\infty} C_{l, n}(t)\left(\varphi^{[n]}(t)\right)^{l}+G(t) .
$$

where $G \in P A P(\mathbb{R}, \mathbb{R})$ and $C_{1,1}(t)<0$. It is easy to see that the linear equation

$$
x^{\prime}(t)=C_{1,1}(t) x(t)
$$

admits an exponential dichotomy on $\mathbb{R}$, by Theorem 2.3 in [15], we know that (2.2) has exactly one solution

$$
x_{\varphi}(t)=\int_{-\infty}^{t} e^{\int_{s}^{t} C_{1,1}(u) d u}\left(\sum_{n=2}^{k} C_{1, n}(s) \varphi^{[n]}(s)+\sum_{n=1}^{k} \sum_{l=2}^{\infty} C_{l, n}(s)\left(\varphi^{[n]}(s)\right)^{l}+G(s)\right) d s
$$

in $X$.

Let $A: B_{C}(M, L) \rightarrow B C(\mathbb{R}, \mathbb{R})$ be defined by

$$
(A \varphi)(t)=\int_{-\infty}^{t} e^{\int_{s}^{t} C_{1,1}(u) d u}\left(\sum_{n=2}^{k} C_{1, n}(s) \varphi^{[n]}(s)+\sum_{n=1}^{k} \sum_{l=2}^{\infty} C_{l, n}(s)\left(\varphi^{[n]}(s)\right)^{l}+G(s)\right) d s, t \in \mathbb{R} .
$$

Note $A: B_{P A P}(M, L) \rightarrow P A P(\mathbb{R}, \mathbb{R})$.

Lemma 2.1. For any $\varphi, \psi \in B_{C}(M, L), t_{1}, t_{2} \in \mathbb{R}$, the following inequality hold,

$$
\left\|\varphi^{[n]}-\psi^{[n]}\right\| \leq \sum_{j=0}^{n-1} L^{j}\|\varphi-\psi\|, n=1,2, \ldots
$$

Proof. It can be obtained by direct calculation by the definition of $B_{C}(M, L)$.

Lemma 2.2. Operator $A: B_{C}(M, L) \rightarrow C(\mathbb{R}, \mathbb{R})$ is continuous.

Proof. Let $\varphi_{j} \rightarrow \varphi_{0}$ as $j \rightarrow \infty$ for $\varphi_{j} \in B_{C}(M, L), j \in \mathbb{N}_{0}=\mathbb{N} \cup\{0\}$ uniformly on any compact interval [- $\left.m, m\right]$, $m \in \mathbb{N}$ of $\mathbb{R}$. Set

$$
h_{j}(s)=\sum_{n=2}^{k} C_{1, n}(s) \varphi_{j}^{[n]}(s)+\sum_{n=1}^{k} \sum_{l=2}^{\infty} C_{l, n}(s)\left(\varphi_{j}^{[n]}(s)\right)^{l}+G(s), j \in \mathbb{N}_{0} .
$$


Then $h_{j} \rightarrow h_{0}$ uniformly on $[-m, m]$. Next we have

$$
\left|e^{\int_{s}^{-m} C_{1,1}(u) d u} h_{j}(s)\right| \leq\left(M \sum_{n=2}^{k} C_{1, n}^{+}+\sum_{n=1}^{k} \sum_{l=2}^{\infty} C_{l, n}^{+} M^{l}+G^{+}\right) e^{-C_{1,1}^{+}(s+m)}, \quad s \in[-\infty,-m] .
$$

Since

$$
\int_{-\infty}^{-m} e^{-C_{1,1}^{+}(s+m)} d s=-\frac{1}{C_{1,1}^{+}}<\infty,
$$

we can apply the Lebesgue dominated convergence theorem to obtain $\left(A \varphi_{j}\right)(-m) \rightarrow\left(A \varphi_{0}\right)(-m)$. From (2.2),

$$
x_{j}^{\prime}(t)-x_{0}^{\prime}(t)=C_{1,1}(t)\left(x_{j}(t)-x_{0}(t)\right)+h_{j}(t)-h_{0}(t)
$$

for $x_{j}(t)=\left(A \varphi_{j}\right)(t), k \in \mathbb{N}_{0}$. Integrating the both sides of (2.5) from $-m$ to $t$, we have

$$
x_{j}(t)-x_{0}(t)=x_{j}(-m)-x_{0}(-m)+\int_{-m}^{t} C_{1,1}(s)\left(x_{j}(s)-x_{0}(s)\right) d s+\int_{-m}^{t}\left(h_{j}(s)-h_{0}(s)\right) d s
$$

and

$$
\left|x_{j}(t)-x_{0}(t)\right| \leq\left|x_{j}(-m)-x_{0}(-m)\right|+2 m\left\|h_{j}-h_{0}\right\|_{m}-C_{1,1}^{+} \int_{-m}^{t}\left|x_{j}(s)-x_{0}(s)\right| d s
$$

for any $t \in[-m, m]$. Then Gronwall's inequality implies

$$
\left\|x_{j}-x_{0}\right\|_{m} \leq\left(\left|x_{j}(-m)-x_{0}(-m)\right|+2 m\left\|h_{j}-h_{0}\right\|_{m}\right) e^{-2 C_{1,1}^{+} m},
$$

which means

$$
\left\|A \varphi_{j}-A \varphi_{0}\right\|_{m} \leq\left(\left|A \varphi_{j}(-m)-A \varphi_{0}(-m)\right|+2 m\left\|h_{j}-h_{0}\right\| m\right) e^{-2 C_{1,1}^{+} m} .
$$

Hence $A \varphi_{j}(t) \rightarrow A \varphi_{0}(t)$ uniformly on $t \in[-m, m]$. Since $m \in \mathbb{N}$ is arbitrarily, we get $A \varphi_{j} \rightarrow A \varphi_{0}$ in $C(\mathbb{R}, \mathbb{R})$, i.e., $A: B_{C}(M, L) \rightarrow C(\mathbb{R}, \mathbb{R})$ is continuous. This proves the continuity of $A$.

Of course, then $A: B_{P A P}(M, L) \rightarrow C(\mathbb{R}, \mathbb{R})$ is continuous as well.

Theorem 2.3. Suppose (H) holds, then Eq. (1.1) has a solution

$$
\varphi \in \overline{B_{P A P}(M, L)} \subset C(\mathbb{R}, \mathbb{R})
$$

for the constants $M$ and L satisfy

$$
\begin{gathered}
\sum_{n=1}^{k} \sum_{l=1}^{\infty} C_{l, n}^{+} M^{l}+G^{+} \leq 0, \\
2\left(M \sum_{n=2}^{k} C_{1, n}^{+}+\sum_{n=1}^{k} \sum_{l=2}^{\infty} C_{l, n}^{+} M^{l}+G^{+}\right) \leq L .
\end{gathered}
$$

For any $\varphi \in B_{C}(M, L), t, t_{1}, t_{2} \in \mathbb{R}$, from (2.6) we have

$$
\begin{aligned}
|(A \varphi)(t)| \leq & \sum_{n=2}^{k} C_{1, n}^{+}\left|\int_{-\infty}^{t} e^{\int_{s}^{t} C_{1,1}(u) d u} \varphi^{[n]}(s) d s\right|+\sum_{n=1}^{k} \sum_{l=2}^{\infty} C_{l, n}^{+}\left|\int_{-\infty}^{t} e^{\int_{s}^{t} C_{1,1}(u) d u}\left(\varphi^{[n]}(s)\right)^{l} d s\right| \\
& +\left|\int_{-\infty}^{t} e^{\int_{s}^{t} C_{1,1}(u) d u} G(s) d s\right|
\end{aligned}
$$




$$
\begin{aligned}
& \leq-\frac{M}{C_{1,1}^{+}} \sum_{n=2}^{k} C_{1, n}^{+}-\frac{1}{C_{1,1}^{+}} \sum_{n=1}^{k} \sum_{l=2}^{\infty} C_{l, n}^{+} M^{l}-\frac{G^{+}}{C_{1,1}^{+}} \\
& \leq \quad M .
\end{aligned}
$$

Without loss of generality, assume $t_{2} \geq t_{1}$, using (2.7)

$$
\begin{aligned}
\left|(A \varphi)\left(t_{2}\right)-(A \varphi)\left(t_{1}\right)\right| \leq & \mid \int_{-\infty}^{t_{2}} e^{\int_{s}^{t_{2}} C_{1,1}(u) d u}\left(\sum_{n=2}^{k} C_{1, n}(s) \varphi^{[n]}(s)+\sum_{n=1}^{k} \sum_{l=2}^{\infty} C_{l, n}(s)\left(\varphi^{[n]}(s)\right)^{l}\right. \\
& +G(s)) d s-\int_{-\infty}^{t_{1}} e^{\int_{s}^{t_{1}} C_{1,1}(u) d u}\left(\sum_{n=2}^{k} C_{1, n}(s) \varphi^{[n]}(s)\right. \\
& \left.+\sum_{n=1}^{k} \sum_{l=2}^{\infty} C_{l, n}(s)\left(\varphi^{[n]}(s)\right)^{l}+G(s)\right) d s \mid \\
\leq & \sum_{n=2}^{k}\left[\left|\int_{-\infty}^{t_{1}} e^{\int_{s}^{t_{1}} C_{1,1}(u) d u}\left(e^{\int_{t_{1}}^{t_{2}} C_{1,1}(u) d u}-1\right) C_{1, n}(s) \varphi^{[n]}(s) d s\right|\right. \\
& \left.+\left|\int_{t_{1}}^{t_{2}} e^{\int_{s}^{t_{2}} C_{1,1}(u) d u} C_{1, n}(s) \varphi^{[n]}(s) d s\right|\right] \\
& +\sum_{n=1}^{k} \sum_{l=2}^{\infty}\left[\mid \int_{-\infty}^{t_{1}} e^{\int_{s}^{t_{1}} C_{1,1}(u) d u}\left(e^{\int_{t_{1}}^{t_{2}} C_{1,1}(u) d u}-1\right) C_{l, n}(s)\left(\varphi^{[n]}(s)\right)^{l}\right) d s \mid \\
& \left.+\left|\int_{t_{1}}^{t_{2}} e^{\int_{s}^{t_{2}} C_{1,1}(u) d u} C_{l, n}(s)\left(\varphi^{[n]}(s)\right)^{l} d s\right|\right] \\
& +\left[\left|\int_{-\infty}^{t_{1}} e^{\int_{s}^{t_{1}} C_{1,1}(u) d u}\left(e^{\int_{t_{1}}^{t_{2}} C_{1,1}(u) d u}-1\right) G(s) d s\right|\right. \\
& \left.+\left|\int_{t_{1}}^{t_{2}} e^{\int_{s}^{t_{2}} C_{1,1}(u) d u} G(s) d s\right|\right] \\
\leq & 2 M \sum_{n=2}^{k} C_{1, n}^{+}\left|t_{2}-t_{1}\right|+2 \sum_{n=1}^{k} \sum_{l=2}^{\infty} C_{l, n}^{+} M^{l}\left|t_{2}-t_{1}\right|+2 G^{+}\left|t_{2}-t_{1}\right| \\
& L\left|t_{1}\right| \cdot
\end{aligned}
$$

This shows that $A \varphi \in B_{C}(M, L)$. Hence $A: B_{C}(M, L) \rightarrow B_{C}(M, L)$ and also $A: B_{P A P}(M, L) \rightarrow B_{P A P}(M, L)$. By Lemma 2.2 we get $A: \overline{B_{P A P}(M, L)} \rightarrow \overline{B_{P A P}(M, L)}$. So all conditions of Tikhonov fixed theorem are satisfied for $A$ with $\Omega=\overline{B_{P A P}(M, L)}$ and $X=C(\mathbb{R}, \mathbb{R})$. Thus there exists a fixed point $\varphi$ in $\overline{B_{P A P}(M, L)}$ of $A$. This is equivalent to say that $\varphi$ is a solution of (1.1) in $\overline{B_{P A P}(M, L)}$. This completes the proof.

\section{Uniqueness and stability}

In this section, uniqueness and stability of (1.1) will be proved.

In addition to the assumption of Theorem 2.3, suppose that

$$
\sum_{n=2}^{k} \sum_{j=0}^{n-1} L^{j} C_{1, n}^{+}+\sum_{n=1}^{k} \sum_{l=2}^{\infty} \sum_{j=0}^{n-1} L^{j} C_{l, n}^{+} l M^{l-1}<-C_{1,1}^{+},
$$

then Eq. (1.1) has unique solution in $B_{P A P}(M, L)$. 
For operator $A$ from $B_{P A P}(M, L)$ into $B_{P A P}(M, L)$, where we consider $B_{P A P}(M, L)$ in $B C(\mathbb{R}, \mathbb{R})$. For $\varphi, \psi \in$ $B_{P A P}(M, L)$, by (2.3)and (2.4)

$$
\begin{aligned}
|\varphi(t)-\psi(t)|= & |(A \varphi)(t)-(A \psi)(t)| \\
\leq & \sum_{n=2}^{k}\left|\int_{-\infty}^{t} e^{\int_{s}^{t} C_{1,1}(u) d u} C_{1, n}(s)\left(\varphi^{[n]}(s)-\psi^{[n]}(s)\right) d s\right| \\
& +\sum_{n=1}^{k} \sum_{l=2}^{\infty}\left|\int_{-\infty}^{t} e^{\int_{s}^{t} C_{1,1}(u) d u} C_{l, n}(s)\left(\left(\varphi^{[n]}(s)\right)^{l}-\left(\psi^{[n]}(s)\right)^{l}\right) d s\right| \\
\leq & -\frac{1}{C_{1,1}^{+}}\left(\sum_{n=2}^{k} \sum_{j=0}^{n-1} L^{j} C_{1, n}^{+}+\sum_{n=1}^{k} \sum_{l=2}^{\infty} \sum_{j=0}^{n-1} L^{j} C_{l, n}^{+} l M^{l-1}\right)\|\varphi-\psi\| \\
= & \Gamma\|\varphi-\psi\|,
\end{aligned}
$$

where $\Gamma=-\frac{1}{C_{1,1}^{+}}\left(\sum_{n=2}^{k} \sum_{j=0}^{n-1} L^{j} C_{1, n}^{+}+\sum_{n=1}^{k} \sum_{l=2}^{\infty} \sum_{j=0}^{n-1} L^{j} C_{l, n}^{+} l M^{l-1}\right)$.

Thus

$$
\|\varphi-\psi\| \leq \Gamma\|\varphi-\psi\| .
$$

By (3.8), we know $\Gamma<1$ and the fixed point $\varphi$ must be unique.

The unique solution obtained in Theorem 3.1 depends continuously on the given functions $C_{l, n}(t)$ and $G(t)$, for $l=1,2, \ldots, ; n=1, \ldots, k$.

Proof. Under the assumptions of Theorem 3.1, if any functions $C_{l, n}(t), \widetilde{C}_{l, n}(t)$ and $G(t), \widetilde{G}(t)$ are given, and satisfy (2.6) and (2.7) for $l=1, \ldots, \infty ; n=1, \ldots, k$. Then there are two unique corresponding functions $x(t)$ and $\widetilde{x}(t)$ in $B_{P A P}(M, L)$ such that

$$
\begin{aligned}
x(t) & =(A x)(t) \\
& =\int_{-\infty}^{t} e^{\int_{s}^{t} C_{1,1}(u) d u}\left(\sum_{n=2}^{k} C_{1, n}(s) x^{[n]}(s)+\sum_{n=1}^{k} \sum_{l=2}^{\infty} C_{l, n}(s)\left(x^{[n]}(s)\right)^{l}+G(s)\right) d s
\end{aligned}
$$

and

$$
\begin{aligned}
\widetilde{x}(t) & =(\widetilde{A} \widetilde{x})(t) \\
& =\int_{-\infty}^{t} e^{\int_{s}^{t} \widetilde{C}_{1,1}(u) d u}\left(\sum_{n=2}^{k} \widetilde{C}_{1, n}(s) \widetilde{x}^{[n]}(s)+\sum_{n=1}^{k} \sum_{l=2}^{\infty} \widetilde{C}_{l, n}(s)\left(\widetilde{x}^{[n]}(s)\right)^{l}+\widetilde{G}(s)\right) d s .
\end{aligned}
$$

Then

$$
\|x-\widetilde{x}\|=\|A x-\widetilde{A} \widetilde{x}\| \leq\|A x-A \widetilde{x}\|+\|A \widetilde{x}-\widetilde{A} \widetilde{x}\|,
$$

i.e.,

$$
\|x-\widetilde{x}\| \leq \frac{\|A \widetilde{x}-\widetilde{A} \widetilde{x}\|}{1-\Gamma} .
$$

Note

$$
\begin{aligned}
|(A \widetilde{x})(t)-(\widetilde{A} \widetilde{x})(t)| \leq & \mid \int_{-\infty}^{t} e^{\int_{s}^{t} C_{1,1}(u) d u}\left(\sum_{n=2}^{k} C_{1, n}(s) \widetilde{x}^{[n]}(s)+\sum_{n=1}^{k} \sum_{l=2}^{\infty} C_{l, n}(s)\left(\widetilde{x}^{[n]}(s)\right)^{l}+G(s)\right) d s \\
& -\int_{-\infty}^{t} e^{\int_{s}^{t} \widetilde{C}_{1,1}(u) d u}\left(\sum_{n=2}^{k} \widetilde{C}_{1, n}(s) \widetilde{x}^{[n]}(s)+\sum_{n=1}^{k} \sum_{l=2}^{\infty} \widetilde{C}_{l, n}(s)\left(\widetilde{x}^{[n]}(s)\right)^{l}+\widetilde{G}(s)\right) d s \mid
\end{aligned}
$$




$$
\begin{aligned}
& \leq \mid \int_{-\infty}^{t}\left(e^{\int_{s}^{t} C_{1,1}(u) d u}-e^{\int_{s}^{t} \widetilde{C}_{1,1}(u) d u}\right)\left(\sum_{n=2}^{k} C_{1, n}(s) \widetilde{x}^{[n]}(s)+\sum_{n=1}^{k} \sum_{l=2}^{\infty} C_{l, n}(s)\left(\widetilde{x}^{[n]}(s)\right)^{l}\right. \\
& +G(s)) d s\left|+\sum_{n=2}^{k}\right| \int_{-\infty}^{t} e^{\int_{s}^{t} \widetilde{C}_{1,1}(u) d u}\left(C_{1, n}(s)-\widetilde{C}_{1, n}(s)\right) \widetilde{x}^{[n]}(s) d s \mid \\
& +\sum_{n=1}^{k} \sum_{l=2}^{\infty}\left|\int_{-\infty}^{t} e^{\int_{s}^{t} \widetilde{C}_{1,1}(u) d u}\left(C_{l, n}(s)-\widetilde{C}_{l, n}(s)\right)\left(\widetilde{x}^{[n]}(s)\right)^{l} d s\right| \\
& +\left|\int_{-\infty}^{t} e^{\int_{s}^{t} \widetilde{C}_{1,1}(u) d u}(G(s)-\widetilde{G}(s)) d s\right| \\
& \leq \frac{1}{C_{1,1}^{+} \widetilde{C}_{1,1}^{+}}\left(M \sum_{n=2}^{k} C_{1, n}^{+}+\sum_{n=1}^{k} \sum_{l=2}^{\infty} C_{l, n}^{+} M^{l}+G^{+}\right)\left\|C_{1,1}-\widetilde{C}_{1,1}\right\| \\
& -\frac{M}{\widetilde{C}_{1,1}^{+}} \sum_{n=2}^{k} \|\left(C_{1, n}-\widetilde{C}_{1, n}\left\|-\frac{1}{\widetilde{C}_{1,1}^{+}} \sum_{n=1}^{k} \sum_{l=2}^{\infty} M^{l}\right\| C_{l, n}-\widetilde{C}_{l, n} \|\right. \\
& -\frac{1}{\widetilde{C}_{1,1}^{+}}\|G-\widetilde{G}\| \\
& \leq \Delta\left(\sum_{l=1}^{\infty} \sum_{n=1}^{k}\left\|C_{l, n}-\widetilde{C}_{l, n}\right\|+\|G-\widetilde{G}\|\right),
\end{aligned}
$$

where

$$
\Delta=-\frac{1}{\widetilde{C}_{1,1}^{+}} \max \left\{-\frac{1}{C_{1,1}^{+}}\left(M \sum_{n=2}^{k} C_{1, n}^{+}+\sum_{n=1}^{k} \sum_{l=2}^{\infty} C_{l, n}^{+} M^{l}+G^{+}\right), 1, M, M^{2}, M^{3}, \ldots\right\} .
$$

Using (3.9) and (3.10), we have

$$
\|x-\widetilde{x}\| \leq \frac{\Delta}{1-\Gamma}\left(\sum_{l=1}^{\infty} \sum_{n=1}^{k}\left\|C_{l, n}-\widetilde{C}_{l, n}\right\|+\|G-\widetilde{G}\|\right) .
$$

This completes the proof.

\section{Example}

In this section, an example is provided to illustrate that the assumptions of Theorem 2.3 do not self-contradict.

Example 4.1. Now, we will show that the conditions in Theorem 2.3 do not self-contradict. Consider the following equation:

$$
\begin{aligned}
x^{\prime}(t)= & -\sum_{l=1}^{\infty} \frac{|\sin t+\sin \sqrt{3} t|+8}{100^{l}}(x(t))^{l}+\sum_{l=1}^{\infty} \frac{|\cos t+\cos \sqrt{5} t|+1}{100^{l}}\left(x^{[2]}(t)\right)^{l} \\
& +\frac{1}{100}(\sin t+\sin \sqrt{2} t),
\end{aligned}
$$

where $C_{l, 1}(t)=-\frac{|\sin t+\sin \sqrt{3} t|+8}{100^{l}}, C_{l, 2}(t)=\frac{|\cos t+\cos \sqrt{5} t|+1}{100^{l}}$, in particular $C_{1,1}(t)=-\frac{|\sin t+\sin \sqrt{3} t|+8}{100}, G(t)=$ $\frac{1}{100}(\sin t+\sin \sqrt{2} t)$. Take $M=1, L=1$, a simple calculation yields

$$
-\frac{1}{10} \leq C_{1,1}^{+} \leq-\frac{2}{25}, C_{s, 1}^{+} \leq \frac{10}{100^{l}}, C_{l, 2}^{+} \leq \frac{3}{100^{l}}, G^{+} \leq \frac{1}{50}, s=2,3, \ldots, l=1,2, \ldots
$$




$$
\sum_{n=1}^{2} \sum_{l=1}^{\infty} C_{l, n}^{+} M^{l}+G^{+} \leq-\frac{71}{2475}<0
$$

and

$$
2\left(M C_{1,2}^{+}+\sum_{n=1}^{2} \sum_{l=2}^{\infty} C_{l, n}^{+} M^{l}+G^{+}\right) \leq \frac{254}{2475}<1=L .
$$

then (2.6) and (2.7) are satisfied. By Theorem 2.3, equation (4.11) has a solution in $B_{P A P}(1,1)$.

Acknowledgement: This work was partially supported by the National Natural Science Foundation of China (Grant No. 11501069, 11671061), Science and Technology Research Program of Chongqing Municipal Education Commission (Grant No. KJQN201800502, KJQN201900525), Foundation of youth talent of Chongqing Normal University (02030307-00039)

\section{References}

[1] R. Bellman, K. Cooke, Differential-Difference Equations, Acadmic Press, 1963.

[2] J. Hale, Theory of Functional Differential Equations, New York, Springer Verlag, 1977.

[3] K. L. Cooke, Functional differential systems: some models and perturbation problems, in: Proceedings of the International Symposium on Differential Equations and Dynamical Systems, Puerto Rico, 1965, New York, Academic Press, 1967.

[4] J.G. Si and X.P. Wang, Smooth solutions of a nonhomogeneous iterative functional differential equation with variable coefficients, J. Math. Anal. Appl., 226(1998), 377-392.

[5] J. Liu and J.G. Si, Analytic solutions for a class of differential equation with delays depending on state, Appl. Math. and Comput., 186(2007), 261-270.

[6] H.Y. Zhao and M. Fečkan, Periodic solutions for a class of differential equation with delays depending on state, Math. Commun. 23 (2018), 29-42.

[7] T.A. Burton, Stability by fixed point theory for functional differential equations, Dover, New York, 2006.

[8] E. Eder, The functional differential equation $x^{\prime}(t)=x(x(t))$, J. Differential Equations 54 (1984), 390-400.

[9] M. Fečkan, On a certain type of functional differential equations, Math. Slovaca 43 (1993), 39-43.

[10] S. Staněk, On global properties of solutions of functional differential equation $x^{\prime}(t)=x(x(t))+x(t)$, Dyn. Syst. Appl. 4(2) (1995), 263-278.

[11] X. P. Wang and J. G. Si, Analytic solutions of an iterative functional differential equation, J. Math. Anal. Appl. 262(2) (2001), 490-498.

[12] F. Chérif, Existence and global exponential stability of pseudo almost periodic solution for SICNNs with mixed delays, $J$. Appl. Math. Comput. 39(2) (2012), 235-251.

[13] E. Ait Dads and M. K. Ezzinbi, Pseudo almost periodic solutions of some delay differential equations, J. Math. Anal. Appl. 201(3) (1996), 840-850.

[14] L. Duan and L. Huang, Pseudo almost periodic dynamics of delay Nicholson's blowflies model with a linear harvesting term, Math. Methods Appl. Sci. 38(6) (2015), 1178-1189.

[15] C. Zhang, Pseudo almost periodic solutions of some differential equations. II., J. Math. Anal. Appl. 192 (1995), 543-561.

[16] H. Zhang, New results on the positive pseudo almost periodic solutions for a generalized model of hematopoiesis, Electron. J. Qual. Theory Differ. Equ. 2014 (1984), 1-10.

[17] B. Liu and C. Tunç, Pseudo almost periodic solutions for a class of first order differential iterative equations, Appl. Math. Lett. 40 (2015), 29-34.

[18] W. Wang, Positive pseudo almost periodic solutions for a class of differential iterative equations with biological background, Appl. Math. Lett. 46 (2015), 106-110.

[19] H.Y. Zhao and M. Fečkan, Pseudo almost periodic solutions of an iterative equation with variable coefficients, Miskolc Mathematical Notes 18 (2017), 515-524.

[20] J. Andres and A.M. Bersani, Almost-periodicity problem as a fixed-point problem for evolution inclusions, Topol. Methods Nonlinear Anal. 18(2) (2001), 337-349.

[21] C. Zhang, Almost periodic type functions and ergodicity. Dordrecht: Kluwer Academic Publishers; Beijing: Science Press, 2003. 